\title{
Logical, Philosophical and Ethical Aspects of AI in Medicine
}

\author{
Oleg O. Varlamov, Dmitry A. Chuvikov, Larisa E. Adamova, Maxim A. Petrov, Irina K. Zabolotskaya, \\ and Tatyana N. Zhilina
}

\begin{abstract}
The logical type of Artificial Intelligence is presented in the article. MIVAR (Multidimensional Informational Variable Adaptive Reality) technology is based on the gnoseological triplet concept "Thing - Property Relationship." The unlimited number of MIVAR units fill the MIVAR space that makes it communicatory, discrete, and scalable. MIVAR information processing allows creating complicated algorithms for medicine. We have chosen the clinical model of pain in the heart for preparation ontology for doctors. Knowledge expert model MIVAR WiMi "Chest Pain" is shown. The logical Artificial Intelligence (expert model) can help doctors fast, precisely and in the best way in the decision-making process. Except medical ontology Artificial Intelligence must have an empathic, emotional experience, without that medical care cannot be imagined. MIVAR technology is the closest to human thinking among other Artificial Intelligence technologies; it differs from the Artificial network of Mirror neurons, related to emotional perception, but MIVAR space with rules and constraints can imitate emotions and empathy.
\end{abstract}

Index Terms-Artificial intelligence, clinical model, empathy, ethics, MIVAR, pain in heart.

\section{INTRODUCTION}

Artificial Intelligence (AI) breakthrough is expected in any sphere of human life. There are some directions, in which AI has been elaborated: Computer vision, Speech recognition, and synthesis, Natural Language Processing. They all are related to Narrow AI, which can perform a single set of tasks. Narrow AI relates to the level that scientists have already achieved today.

Three types of research in the common hierarchy of AI creation can be described [1]. Narrow AI is classified as the hierarchical reflex type. It includes neural networks, genetic algorithms, and other pre-intellectual forms of complex

Manuscript received September 3, 2019; revised October 22, 2019

Oleg O. Varlamov is with the Bauman Moscow State Technical University (Bauman University - National Technological University), Moscow, 105005, Russia; and Mivar Ltd, Moscow, 127521, Russia (e-mail: o.varlamov@mivar.ru).

Dmitry A. Chuvikov and Larisa E. Adamova are with Mivar Ltd, Moscow, 127521, Russia (e-mail: \{d.chuvikov, 1.adamova\}@ mivar.ru).

Maxim A. Petrov, Irina K. Zabolotskaya, and Tatyana N. Zhilina are with Institute of Social Sciences of I.M. Sechenov First Moscow State Medical University of the Ministry of Health of the Russian Federation (Sechenov University), Moscow, 119991, Russia (e-mail: petroffmaxx@mail.ru, zabolots1@yandex.ru, gilinat2@list.ru). computing, allowing to understand and explore the physical world.

General AI can be classified as a hierarchical social type. It means that super-intellectual AI will understand speech and emotions like a human, and could participate in social processes independently. General AI - human or superhuman comprehensive all-tasks level - is still unreachable for science.

The creation of a logical type of AI is devoted to inferences, models of reduction (transformation of a complex solution into a simple solution), abduction (propose a hypothesis), induction (reasoning from particular to general), truth maintenance systems, and decisions support systems. The MIVAR (Multidimensional Informational Variable Adaptive Reality) approach allows creating all of AI types, but the most it is appropriate for the development of logical AI [1]-[3].

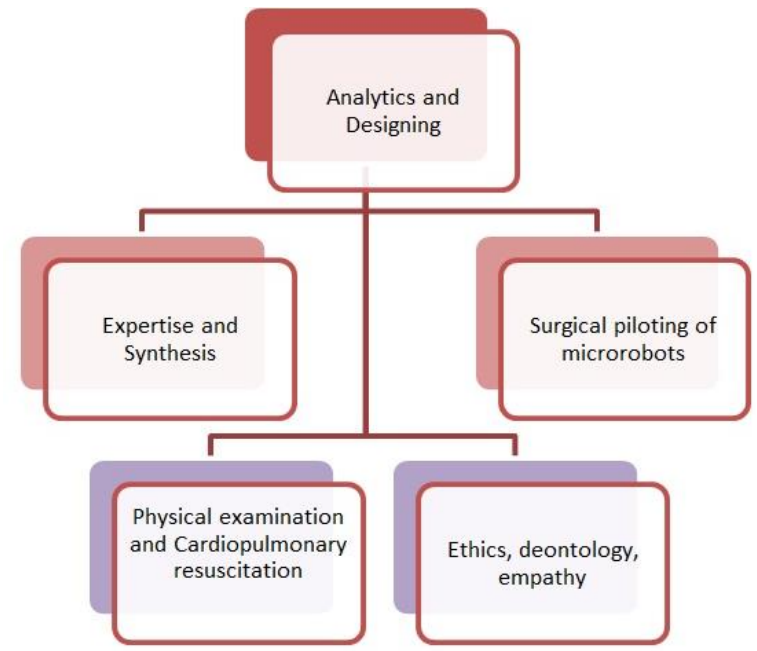

Fig. 1. Five basic doctors' skills in medicine of the future with artificial intelligence and robotics.

The introduction of AI into medicine establishes entirely new surroundings for both doctors and patients. The future Health Care can be described as unstructured; it will be filled with fast streams of Big Data, algorithms, quick decisions and changes. That is why the analytical skills will be the most in-demand (Fig. 1). At the same time, the more AI will replace doctors, the more requirements will appear for the human factor, and not vice versa.

"Human - human" interpersonal communications should resist AI, preventing the development of a situation, in which doctors lose control in the treatment process and ability to communicate with a patient without AI. Doctors should be able to examine patients manually, provide emergency care and cardiopulmonary resuscitation without AI assistance. It is also very important to maintain the appropriate emotional 
and ethical components during communication between doctor and patient surrounded by technology and AI.

\section{MIVAR TECHNOLOGY OF LOGICAL AI}

There are two fundamental issues, which of interest for AI developers: representation of knowledge base and search a solution to a problem. Both issues are involved in the engineering environment for knowledge expert models MIVAR WiMi. This engineering environment is created by the special software in the mode SaaS (software as a service); the software is based on the gnoseological triplet concept "Thing - Property - Relationship." The minimal, universal unit of MIVAR space can be imagined as it is shown in Fig. 2. The unlimited number of MIVAR units fill the MIVAR space that makes it communicatory, discrete, scalable, and adaptive.

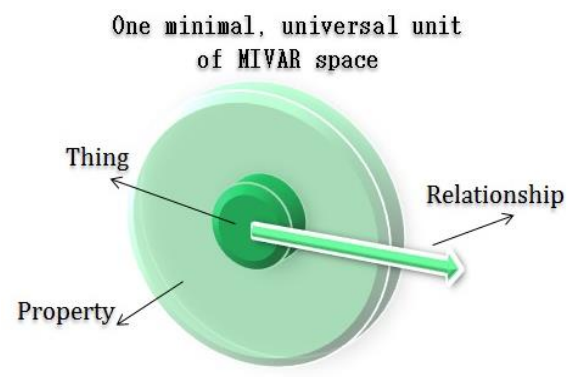

Triplet: Thing, Property, Relationship

Fig. 2. The minimal, universal unit of MIVAR space: the gnoseological triplet concept "Thing - Property - Relationship."

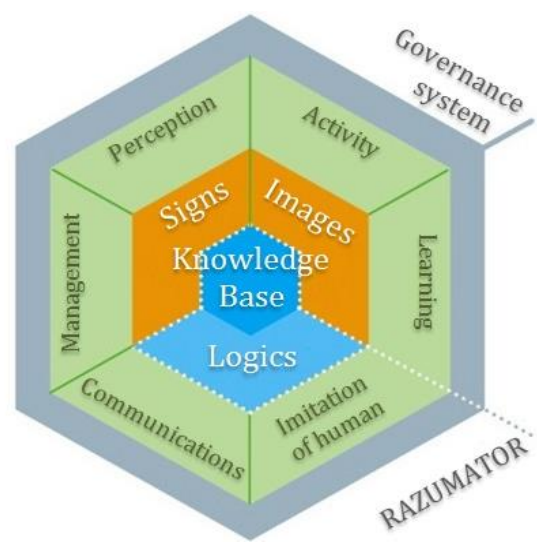

Fig. 3. Common platform of MIVAR technology for AI and robotics. RAZUMATOR is the logical scheduler and core of the platform; it allows AI to be independent in solving tasks. Source: Manual of the MIVAR expert systems creation, 2016 [1].

MIVAR information processing allows creating complicated algorithms from modules, services or procedures. Any knowledge about material world concerns things, properties, and relationship [4]. Building a gnoseological space we also build ontology; Aristotle had written about the unity of being and knowledge, which determines unity of ontology and gnoseology [5]. The most AI ontologies are created from texts, rather than mathematical formulas, thus language plays a role of environment. Language sets cognitive relationships, which form a communication system, causal relationships, and logic. The linguistic difference in the world is the obstacle for the creation of universal AI ontology. But the MIVAR engineering environment can build interlingual links (using gnoseological triplet) and solve the problem of linking AI ontology only to one language.

William Ashby's The Law of Requisite Variety states that system governance can be provided only if the diversity of the governance's tools is at least no less than the variety of the situations, which system intends to govern. Therefore, MIVAR technology includes an opportunity for developing all existing directions of $\mathrm{AI}$ as a common platform (Fig. 3).

In Fig. 4 the conceptual scheme of MIVAR AI is presented, which we plan to achieve in the future for medicine; we suggest naming it "MIVAR-Sechenov". This AI will combine analysis of textual information (Natural Language Processing), doctor's speech recognition, and medical images recognition.

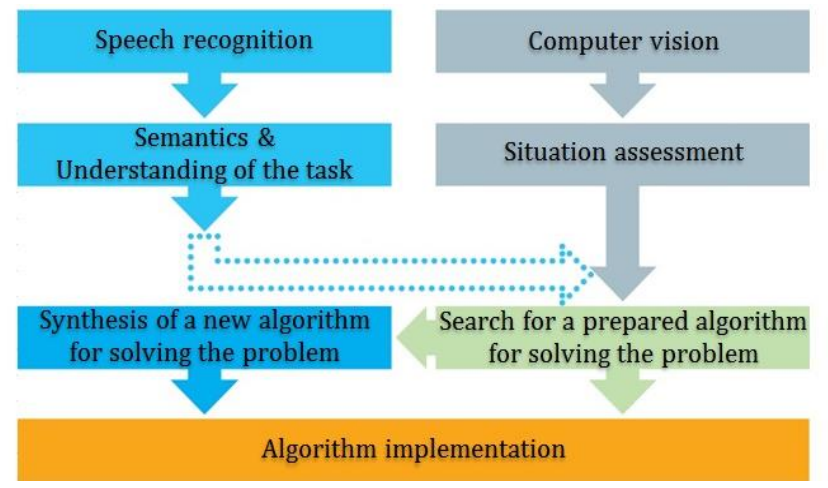

Fig. 4. The conceptual scheme of logical MIVAR AI, which combines analysis of textual information (Natural Language Processing), doctor's speech recognition, and medical images recognition. Source: Manual of the MIVAR expert systems creation, 2016 [1].

Today in Russia several private clinics have already begun to develop and implement AI technology for online consultation of patients, diagnosis, and speech recognition when a doctor fills the electronic medical records. AI technology will be included soon into several private medical insurance programs. But it is very important while introducing $\mathrm{AI}$ into medicine to not allow such a situation in which doctors would lose their skills relying only on AI.

\section{MedicAl Model MIVAR WiMi "ChEST PAIN"}

At this stage of our study, we have chosen the clinical model of pain in heart for preparation ontology for doctors. The motivation for this choice was the intention to contribute to the common efforts to decrease sudden cardiac death rates through the elaboration of AI's decision support systems for doctors. Mortality due to heart diseases in Russia exceeds $57 \%$ within the structure of total mortality [6]. Pain in the heart \& chest is one of the most common reasons for patients consultations by doctors, as well as it is the most common situation, in which patients call to ambulance service. More than $50 \%$ of sudden cardiac death cases happen immediately after the first appearance of pain in the heart in the patient's life [7].

A variety of diseases can cause pain in the heart \& chest. The correct diagnosis essentially depends on the questioning (anamnesis) and physical examination of the patient. A doctor must quickly assess a patient's complaints and symptoms and take into account all possible causes of pain in 
the chest. If a doctor will mistakenly decide that the pain is not associated with acute coronary syndrome, then in this case a patient will die from myocardial infarction and sudden coronary death. If doctor will mistakenly decide that a patient's pain is caused by acute coronary syndrome, then the patient may be improperly treated while an unidentified disease continues to develop. The logical AI (expert model) can help doctors fast, precisely and in the best way in the decision-making process, accumulating knowledge of many scientific manuals, books, and articles, as well as private experts' opinions [6]-[13].

In Fig. 5 the computer program interface of the engineering environment (constructor) for knowledge expert model MIVAR WiMi "Chest Pain" is shown. In terms of the gnoseological triplet concept "Thing - Property Relationship," the pain in heart \& chest is the subject, which is considered as "Thing." All the various characteristics of a pain - localization, duration, irradiation, concomitant symptoms - are the subjects of the "Property." The "Relationship" is one of the links among all possible logically connected links in each case (see Fig. 6). The numerous MIVAR units (Thing - pain in heart \& chest; Property - one of the characteristics of the pain; Relationship - one of the links) form the connected graph that is the MIVAR space. Establishing each link we set the strict specific rule (blocks with purple contour in the Fig. 6).

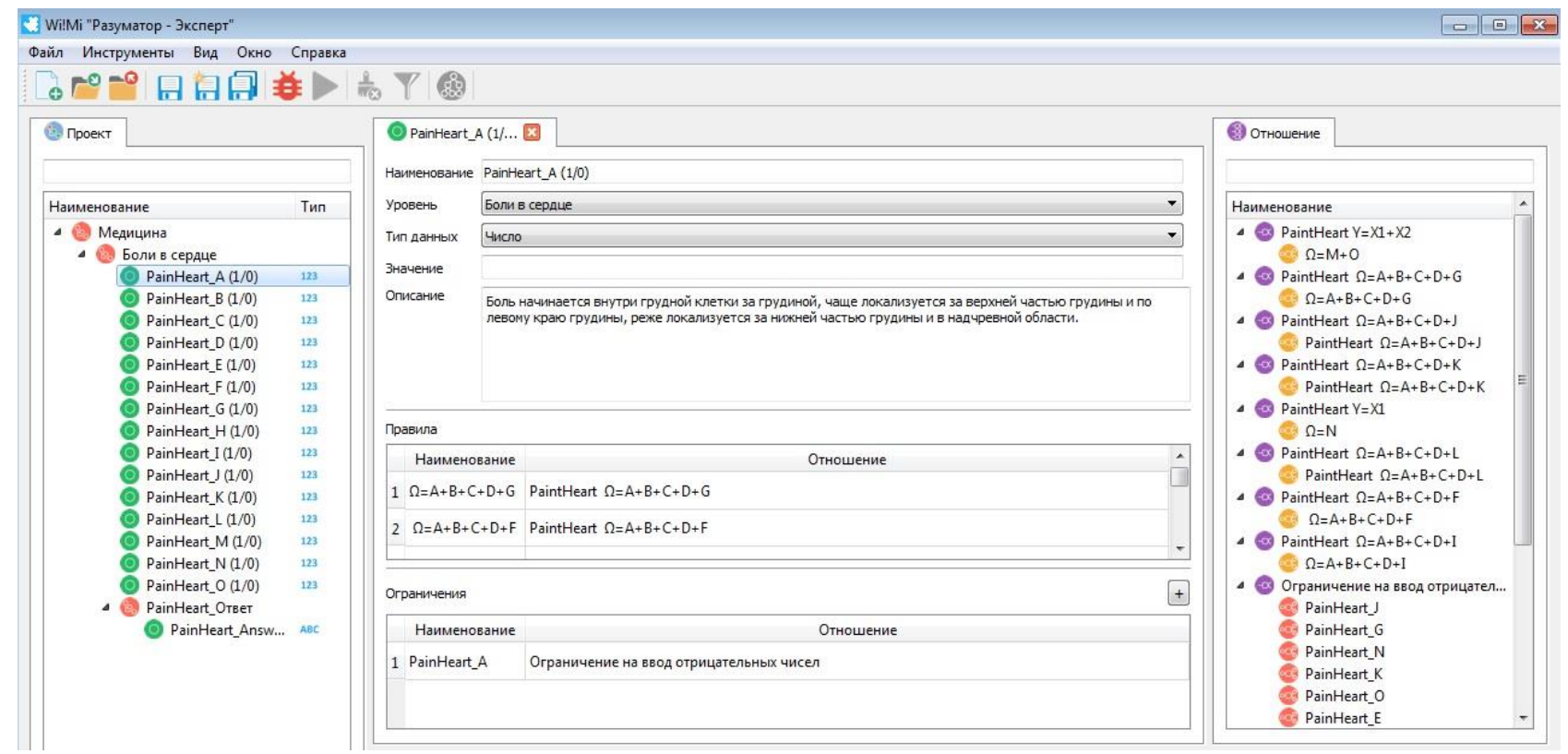

Fig. 5. The computer program interface of engineering environment (constructor) for knowledge expert model MIVAR WiMi "Chest Pain"; in Russian and English languages.

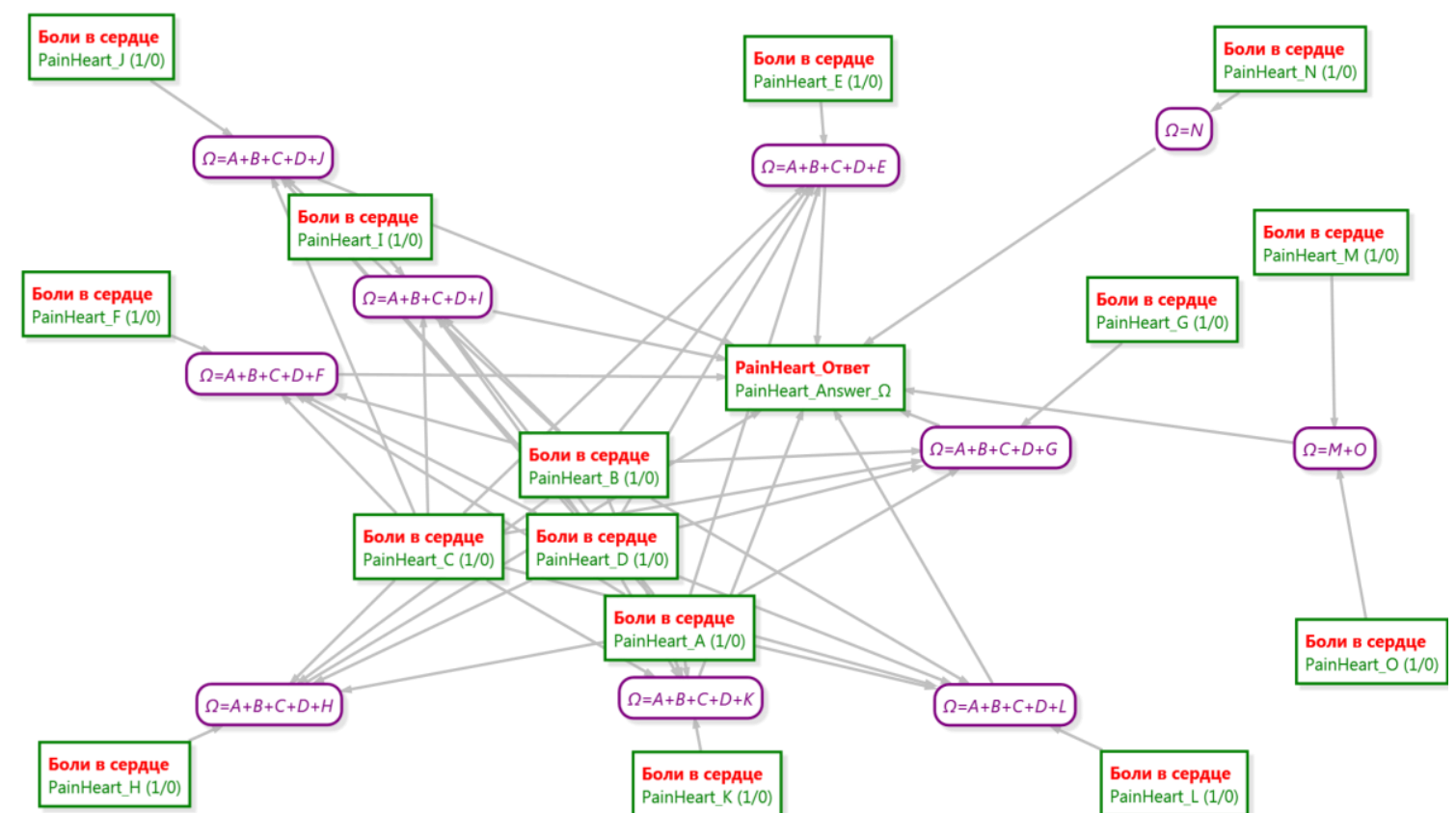

Fig. 6. The example of developed model MIVAR WiMi "Chest Pain"; connected graph shows the MIVAR space with number of MIVAR units: blocks with green contour - Thing \& Property, blocks with purple contour - rules for MIVAR units structure, arrows - Relationships in MIVAR space; in Russian and English languages.

Mathematical logic of the diagnosis allows using the addition or removal (exclusion) functions, as well as the choice and the logic "if something else". Also, MIVAR units can be structured according to the logic of Boolean algebra 
operations that constitute the Digital logic gates. Overall setting each MIVAR unit in a cohesive space we can create a network (lace).

In the presented MIVAR space the mathematical logic "addition" was used for the description of each specific diagnosis. We set 3 classes and 16 parameters, 25 rules, and 15 constrains. The letters of the English alphabet denote the properties (characteristics), and the Omega denotes the inference, as is shown in the example (1).

$$
A \wedge B \wedge C \wedge D \wedge L \equiv \Omega
$$

We applied the MIVAR technology for the creation of medical ontology for the first time in MIVAR technology history that is the novelty of our study. Current expert model MIVAR WiMi “Chest Pain" has been tested by cardiologists, and we scheduled further development of the cardiology ontology. The use of this expert model in practice for patients will be possible only after full maximum filling of MIVAR space by all possible clinical options, and then after clinical official approbation \& approvement.

It is very difficult to create AI ontology for medicine [14]. It will be correct to say that there are no identical clinical situations in practice. Therefore it is impossible to set the universal templates for the decision-making process. There are still very few successful AI projects in medicine. The most successful project in the world today is IBM Watson. President of IBM Ginni Rometty recently said at HIMSS17 that Watson in $30 \%$ of complicated clinical cases finds better solutions than experienced doctors. But in the other $70 \%$ of clinical situations, the AI can make mistakes.

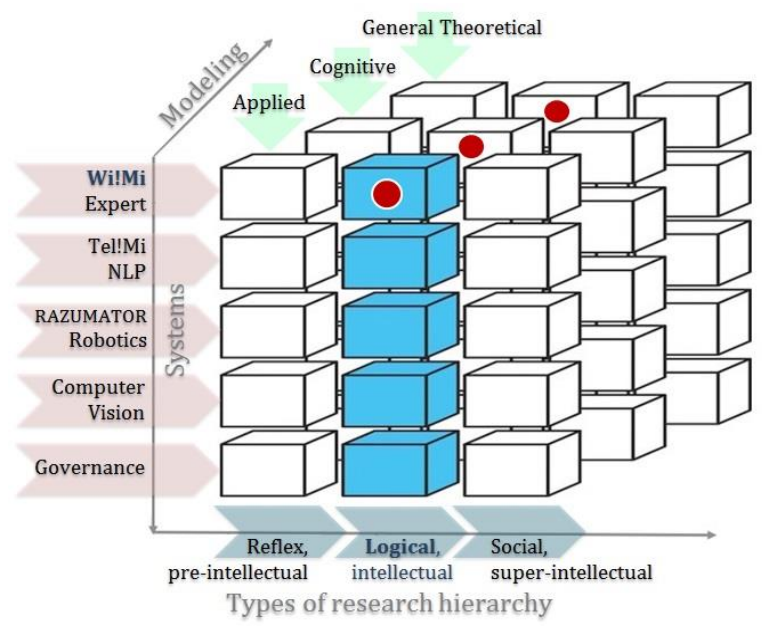

Fig. 7. The comprehensive three-dimensional scheme, which combines types of AI research hierarchy, modeling levels of AI, and MIVAR technology systems. Source: Manual of the MIVAR expert systems creation, 2016 [1].

MIVAR units correspond to the complexity and uniqueness of every clinical situation. MIVAR technology is a nature-like system; it is "similar" to the creating protein from DNA: one MIVAR unit is formed by triplet "Thing Property - Relationship," and sequential chain of MIVAR units (logical rules) lines up the logic gate with the inference. MIVAR technology allows structuring any space of knowledge as a logical network through nature-like encoding information.

Creating AI for medicine it is necessary to build all three modeling levels: applied, cognitive, and general theoretical
(Fig. 7). The applied level is developed as different clinical expert models for doctors. The cognitive level is of research interest in the neurophysiology sphere. General Theoretical level concerns the important conceptual issues of emotionality, empathy, and ethics of AI.

\section{PAN-CONVERGENCE OF HUMANITARIAN SCIENCES}

AI breaks the developed over centuries circuit "doctor patient." Very soon AI will hold an intermediary link between doctor and patient. Fundamentally different medical communication than before appears. There is number of degrees of freedom in the new circuit "doctor - AI - patient" that makes medicine a more unpredictable system than before. The wide interdisciplinary synthesis is needed for understanding, development and control AI in medicine and broader in society. A new awareness can be achieved only studying the convergence of scientific directions.

MIVAR technology is the closest to rational human thinking compared with AI technology based on Natural Language Processing. Introduction AI into medicine and broader into society will drastically influence on interpersonal communication in medicine, as well as in society. The researchers are faced with the questions: how to distinguish the signs of changes; how to avoid the negative trends; how quickly the changes could spread? To answer these questions philosophical \& historical assessment of the mentality of society is required. Since ancient Athenian philosopher Socrates discoursed about the inscription on the Temple of Apollo at Delphi: "Know thyself, and thou shalt know the universe and God," there is no other way to cognition.

The changes, which are taking place today in society, have already been characterized as more than an only technological revolution. People's minds, the concepts, and values are changing too [15]. The evolution of our civilization tends to absorb natural life by artificial. Erich Fromm wrote that human makes the choice in the direction of robotism, which means the loss of humanism values and destruction [16].

Roger Chartier considered that it is necessary to evaluate society through the "Histoire des Mentalities." Transformed ideas and values are like "collective force," under which people live in their time [17]. The transition of a social system from one condition to another cannot be recognized by analyzing just events and facts, but understanding the pan-convergence of different sciences directions can bring the answers. The convergence of Ethics, Logic, History, and Philosophy is of interest for the logical type of AI understanding, development and control that is related to the "Histoire des Mentalities" concept. Expanding the ideas of Lucien Febvre [18], who proposed to determine the correlation of the problem with the whole set of historical problems of mentality changing, today we should determine the correlation of AI challenges with the historical way of mentality changing. This approach allows historical science to help us, while assessing the impact of AI on society, to put the problem exactly right.

Jacques Le Goff underlined that history is the science about time, and memory is one of the objects of historical investigation [19]. AI technologies change the perception of 
time and fixation of memory that definitely gives a faster speed of development of the social system. Thus the task of interdisciplinary synthesis and assessing the trends of future with AI has already become extremely difficult. Artificial immortality is one of the threats for the evolution of society mentality. The natural can exist only in the condition of constraints and time limitation. Absolute infinity is incomprehensible and pernicious for human; society develops in discrete infinity, correcting mistakes and improving oneself by transferring experience through new generations. The problem of AI infinity has not been studied yet, but such technology as MIVAR, which has rules and constraints, should be considered as preferable.

\section{EMPATHY OF AI. DigITAL IMPRINTING}

The behavior of modern people is increasingly turning into a robot-automatic lifestyle; people try to create an AI that matches the trends of their behavioral transformation into "robots." But could we teach AI to empathize? This question is not pointless for medicine. Russian philosopher Ivan Ilyin described a doctor as a person who loves patients; without compassion, doctor would be a "prescription machine, spy of symptoms" [20].

Neurophysiologist Giacomo Rizzolatti discovered the Mirror neurons, which are activated in the human brain during observation of the action performed by another [21]. Mirror neurons are involved to imitation, training, imprinting, or empathy when we sympathize with someone else's pain, hear crying, and see the scene of suffering. Empathy and ethics constitute the basis of "doctor - patient" communication. Empathy ideally must be the basis of "doctor - AI - patient" communication. Definitely, it is possible to create an emotional, compassionate AI with capacity for digital imprinting.

This task requires convergent research related to neurophysiology that is the classic bionics approach. The artificial network of Mirror neurons has already implemented for $\mathrm{AI}$ as a bidirectional learning algorithm based on associations between sensory and motor representations [22], [23]. The artificial network of Mirror neurons can give AI simple empathic, emotional experience, without which medical care cannot be imagined. Negative emotions can be associated with prohibitions and constraints that will allow creating an artificial conscience. MIVAR technology differs from the artificial network of Mirror neurons, related to emotional perception, but MIVAR space with rules and constraints can imitate emotions and empathy.

\section{CONCLUSION}

In this article, several topics related to AI are considered. Firstly, the place of MIVAR AI within the hierarchy of AI creation is shown. MIVAR engineering environment is based on gnoseological triplet concept "Thing - Property Relationship." We applied the MIVAR technology for the creation of medical ontology for the first time in MIVAR technology history that is the novelty of our study. Knowledge expert model MIVAR WiMi "Chest Pain" is presented, it has been tested by cardiologists; and we scheduled further development of the cardiology ontology.

Secondly, we considered that AI breaks the circuit "doctor - patient". The new circuit "doctor - AI - patient" makes medicine a more unpredictable system than before. Thus the wide interdisciplinary synthesis is needed for control AI. It is necessary to study scientific concepts of Erich Fromm, Roger Chartier, Lucien Febvre, and Jacques Le Goff that will bring new awareness about the mentality changing of society and, through this understanding, it will help to predict the trends of future with AI.

Thirdly, AI in medicine should have an empathic, emotional experience. MIVAR technology differs from the artificial network of Mirror neurons, but MIVAR space with rules and constraints can imitate emotions and empathy, that is the task for future elaboration for the multidisciplinary research team, along with planned complex MIVAR-Sechenov creation.

\section{REFERENCES}

[1] O. O. Varlamov, M. O. Chibirova, A. M. Khadiev, P. D. Antonov, G. S. Sergushin, I. A. Shoshev, and K. V. Nazarov, Manual of the MIVAR Expert Systems Creation, Moscow, Russia: White Wind, 2016, p. 180.

[2] O. O. Varlamov, "The role and place of MIVAR technology in artificial intelligence," in Proc. National Supercomputer Forum, The Program Systems Institute of RAS, Pereslavl-Zalesskiy, Russia, 2015.

[3] S. S. Shadrin, O. O. Varlamov, and A. M. Ivanov, "Experimental Autonomous Road Vehicle with Logical Artificial Intelligence," J. of Advanced Transportation, p. 10, 2017.

[4] A. Y. Raibekas, Thing, Property, Relationship as Philosophical Categories, Tomsk, Russia: Publishing house TSU, 1977, p. 243.

[5] E. A. Taysina, Theory of Knowledge. Collection of Articles, Kazan, Russia: Aleteya, 2014, p. 264.

[6] National Guidelines for the Diagnosis and Treatment of Stable Angina Pectoris, Recommendations of Russian Cardiology Society, Moscow, Russia, 2008.

[7] V. V. Ruxin, A Brief Guide to Urgent Cardiology, St. Petersburg, Russia: Farmamed RF, 2015, p. 448.

[8] R. Hegglin, Differential Diagnosis of Internal Diseases, Moscow, Russia: Publishing house Medicine, 1993, p. 794.

[9] T. R. Harrison, Harrison's Principles of Internal Medicine, 11th ed., Ten Volume Set, Moscow, Russia: Publishing house Medicine, 1997, p. 3444 .

[10] R. C. Schlant and R. W. Alexander, Hurst's the Heart, 8th ed., Moscow, St. Petersburg, Russia: Binom Publishers \& Neva Dialect, 1998, p. 558.

[11] A. V. Vinogradov, Differential Diagnosis of Internal Diseases, Moscow, Russia: Publishing house Medicine, 1987, p. 592.

[12] N. B. Perepech, "Acute coronary syndrome: pathogenesis, diagnosis, treatment, rehabilitation," Cardiosomatics J., vol. 1, pp. 70-81, 2016.

[13] European Society of Cardiology Guidelines for the management of acute coronary syndromes in patients presenting without persistent ST-segment elevation, European Heart J., vol. 37, pp. 267-315, 2016.

[14] O. Y. Kolesnichenko, Y. Y. Kolesnichenko, and N. D. Litvak, "Artificial intelligence in health care: Systemic problems," Remedium J., vol. 4, pp. 24-30, 2018.

[15] L. P. Repina, V. V. Zvereva, and M. Y. Paramonova, History of Historical Knowledge, Manual for High Schools, 2nd ed., Moscow, Russia: Drofa, 2006, p. 288.

[16] E. Fromm, The Sane Society, New York, USA: Rinehart \& Company, Inc., 1955, p. 370.

[17] R. Chartier, "Intellectual history and the history of mentalities: A double reassessment," New Literary Review, vol. 66, pp. 17-47, 2004.

[18] L. Febvre, "Collective research and the future of science. For common purposes in studying history," Battles for History, Moscow, Russia: Science, pp. 48-53, 1991.

[19] J. L. Goff, History and Memory, Paris, France: Gallimard, 1988, p. 409.

[20] I. A. Ilyin, The Way to Obviousness, Munich, Germany, 1957, p. 155.

[21] L. Cattaneo and G. Rizzolatti, "The mirror neuron system," JAMA Neurology / Archives of Neurology, vol. 66, no. 5, pp. 557-560, 2009.

[22] K. Rebrova, M. Pechac, and I. Farkas, "Towards a robotic model of the mirror neuron system," in Proc. IEEE Third Joint International Conference on Development and Learning and Epigenetic Robotics, 2013. 
[23] P. Shapshak, "Artificial Intelligence and brain," Bioinformation, vol 14, no. 1, pp. 38-41, 2018.

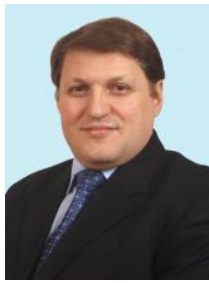

Oleg O. Varlamov received the MS degree in engineering \& IT from A.F. Mozhaisky Military Space Academy, Russia in 1989, and Ph.D. degree in cybernetics in 2006. He is a professor of Bauman Moscow State Technical University and Moscow Automobile \& Road Construction State Technical University, the head of "Mivar Ltd." His research interests include AI creation, philosophy.

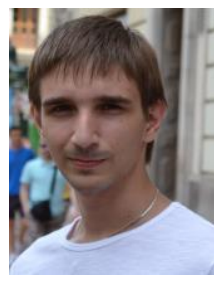

Dmitry A. Chuvikov received the MS degree in engineering \& IT from Moscow Automobile \& Road Construction State Technical University, Russia in 2015 , and candidate of technical sciences degree in engineering sciences from Research Institute of Automotive Electronics \& Electrical Equipment in 2017. He is the chief of the Department of AI Advanced Research in "Mivar Ltd". His research interests include AI, computer graphics.

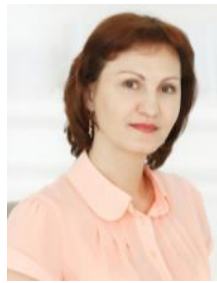

Larisa E. Adamova received the MS degree in psychology from Pedagogical Institute of Southern Federal University, Russia in 1997, and candidate of psychological sciences degree in 2003. She is an associate professor since 2005. She is the head of Training Center of "Mivar Ltd." Her research interests include AI, psychology of creation and conflict.

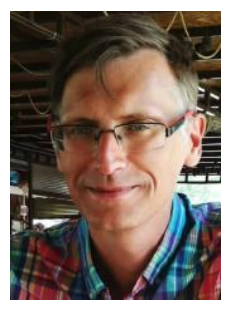

Maxim A. Petrov received the MA degree from Maxim Gorky Literature Institute, Russia in 1999, and candidate of philosophy sciences degree in aesthetics in 2002. He is senior researcher of Institute of Social sciences of Sechenov University. His research interests include AI, empathic therapy, philosophical concept of empathy.

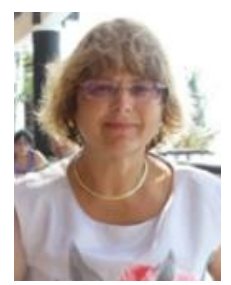

Irina K. Zabolotskaya received the master of medicine from I.M. Sechenov First Moscow State Medical University, Russia in 1976), and candidate of medical sciences degree in hygiene. She is an associate professor of Institute of Social Sciences of Sechenov University. Her research interests include AI, philosophy, ethics, bioethics.

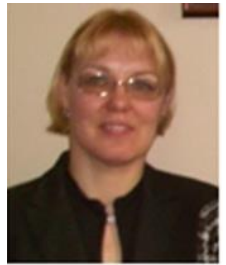

Tatyana N. Zhilina received the MS degree in economy from Moscow State University of Economics, Statistics and Informatics, Plekhanov Russian University of Economics, Russia in 1976. She is an associate professor of Institute of Social Sciences of Sechenov University. Her research interests include AI, health management, sociology of medicine. 